\title{
The Microbial Community in A Chinese Traditional Fermented Suanrou by High Throughput Sequencing
}

\author{
Ping HU* \\ School of Liquor and Food Engineering \\ Guizhou University \\ Guiyang, Guizhou, 550025, P.R. China \\ e-mail: phu1@gzu.edu.cn \\ Jianlong ZHAN \\ School of Liquor and Food Engineering \\ Guizhou University \\ Guiyang, Guizhou, 550025, P.R. China \\ e-mail: phu1@gzu.edu.cn
}

\author{
Yun CHEN \\ School of Liquor and Food Engineering \\ Guizhou University \\ Guiyang, Guizhou, 550025, P.R. China \\ e-mail: phu1@gzu.edu.cn \\ Yulong ZHANG \\ School of Liquor and Food Engineering \\ Guizhou University \\ Guiyang, Guizhou, 550025, P.R. China; \\ e-mail: phu1@gzu.edu.cn
}

\begin{abstract}
Microbial diversity in a Chinese traditional spontaneous fermented sour meat product, Suanrou, was investigated using High-throughput sequencing. A taxonomic analysis was carried out on 1248 OTUs obtained with 454-pyrosequencing of 16S rRNA amplicons of the bacterial microbiota in 3samples of suanrou produced traditionally. Taxonomically, Staphylococcus, Lactobacillus, Cyanobacterium, Tetragenococcus, Weissella, Rickettsiales and Brevibacterium were the mains groups, of which Staphylococci and Lactobacillus were dominant. Microbial diversity in the 3 Suanrou samples was not similar and we concluded that the salt concentration was the main driver for the structure of the microbial community in these Suanrou. Further research aims to develop novel strains for commercial production.
\end{abstract}

Keywords-sour meat; High-throughput sequencing; microbial communities; predominant strains

\section{INTRODUCTION}

A naturally fermented sour meat product, Suanrou, which is consumed in large quantities in the Southwest of China, has been produced by the Dong, Miao and Zhuang minority for more than a thousand years [1]. This fermented sour meat product is produced using a traditional recipe which is characterized by accentuated acidity, a unique flavor and appealing color. This traditional sour meat product maintains its quality (bright color and pleasant flavor) with a shelf life of more than ten years when kept in ceramic jars without refrigeration [1]. It is supposed that there are some special species of microbes contributing to the fermenting process of sour meat.

In recent decades, the strains isolated from these sour meat products, using culture dependent methods, include Bacillus spp., Lactic Acid Bacteria (LAB), Staphylococcus spp. and Micrococcus spp. and yeast [2]. Additionally, some strains of LAB such as Pediococcus pentosaceus, Pediococcus acidilactici, Leuconostoc spp. have been identified [2]; and strains of Staphylococcus spp. [3], Lactobacillus sakei [4] and Weissella hellenica D1501 with potential probiotic properties have been screened from these fermented sour meat products [1].

The developments in the field of molecular biology have increased the understanding of changes in micro-ecology during the production of fermented meat products [5]. The micro-ecology of fermented products is usually investigated using DGGE technology and the association of diverse groups of microorganisms in number of traditional fermented sausage products is available [6, 7] The field of microbial ecology has further advanced with the development of high-throughput sequencing (HTS). HTS improves identification of microbial taxa, including those which are difficult to culture and/or are present in low abundance [8]. The HTS approach has been successfully applied for the monitoring of the microbiota in Italian salami at different ripening stages $[9,10]$ and the assessment of bacterial ecology in sausage production [11].

Many researchers believed that the microorganisms present in traditional fermented meat products are derived from the raw materials or from the environment of manufacturing [12]. In this research, we aim to characterize the bacterial community in traditionally produced sour meat products selected from Southwest China and we aim to investigate this diversity using HTS of 16S rRNA amplicons.

\section{MATERIAL AND METHODS}

\section{A. Sampling Procedures}

Three samples (three replicates) were randomly collected from different farm households in three villages of Dong ethnic minority regions, Guizhou province of China. Suanrou were homemade using traditional method during winter. The raw meat was cut into pieces weighting between 1 and $2 \mathrm{~kg}$ after rubbing with salt and a little Sichuan pepper thoroughly, the meat was put into a vat for curing for 2 to 7 days under room temperature. Then followed addition of hot cooked glutinous rice and mixing 
with appropriate red chopped chili, garlic and rice wine. Then the meat mixtures were laid down into a ceramic jar, the meat compressed tightly while maintaining headspace of the jar, and then the jar neck was covered with wild banana leaves and tightened with straw rope, and finally, covered with plastic cover on top of the jar. The meat mixtures were left to ferment at room temperature for 30 to 60 days. Sour meats were sampled and stored into an ice box, and brought to the lab within 12 hours and kept frozen at $-80^{\circ} \mathrm{C}$ before being subjected to DNA extraction. A description of all the samples is as follows: sample R5 collected from Luanli village with salt added 15\%, meat cured time 2 days and 1 month of fermented time; sample R9 collected from Gaozeng village with salt added 7\%, meat cured time 2 days and 3 months of fermented time; sample R12 collected from Jiasuo village with salt added $15 \%$, meat cured time 7 days and 6 months of fermented time.

\section{B. DNA Extraction and PCR Amplification}

Microbial DNA was extracted from suanrou samples using the E.Z.N.A ${ }^{\circledR}$ bacteria DNA Kit (Omega Bio-tek, Norcross, GA, U.S.) according to manufacturer's protocols. The V1-V3 region of the bacteria 16S ribosomal RNA gene were amplified by PCR $\left(95^{\circ} \mathrm{C}\right.$ for $2 \mathrm{~min}$, followed by 25 cycles at $95^{\circ} \mathrm{C}$ for $30 \mathrm{~s}, 55^{\circ} \mathrm{C}$ for $30 \mathrm{~s}$, and $72^{\circ} \mathrm{C}$ for $30 \mathrm{~s}$ and a final extension at $72^{\circ} \mathrm{C}$ for $5 \mathrm{~min}$ ) using primers $27 \mathrm{~F}$ 5'-AGAGTTTGATCCTGGCTCAG-3' and 533R 5'-TTAC CGCGGCTGCTGGCAC-3', where barcode is an eight-base sequence unique to each sample. PCR reactions were performed in triplicate $20 \mu \mathrm{L}$ mixture containing $4 \mu \mathrm{L}$ of $5 \times$ FastPfu Buffer, $2 \mu \mathrm{L}$ of $2.5 \mathrm{mM}$ dNTPs, $0.8 \mu \mathrm{L}$ of each primer $(5 \mu \mathrm{M}), 0.4 \mu \mathrm{L}$ of FastPfu Polymerase, and 10ng of template DNA.

\section{Pyrosequencing}

Amplicons were extracted from 2\% agarose gels and purified using the AxyPrep DNA Gel Extraction Kit (Axygen Biosciences, Union City, CA, U.S.) according to the manufacturer's instructions and quantified using QuantiFluor $^{\mathrm{TM}}$-ST (Promega, U.S.). Purified amplicons were pooled in equimolar and paired-end sequenced $(2 \times$
250) on a Roche FLX 454 pyrosequencing machine at Majorbio Bio-Pharm Technology Co., Ltd., Shanghai, China according to the standard protocols. The raw reads were deposited into the NCBI Sequence Read Archive (SRA) database.

\section{Processing of Sequencing Data}

Raw fastq files were demultiplexed, quality-filtered using QIIME (version 1.9.1) with the following criteria: (i) The 300 bp reads were truncated at any site receiving an average quality score $<200$ over a 50 bp sliding window, discarding the truncated reads that were shorter than 50bp. (ii) exact barcode matching, 2 nucleotide mismatch in primer matching, reads containing ambiguous characters were removed. (iii) only sequences that overlap longer than 10 bp were assembled according to their overlap sequence. Reads which could not be assembled were discarded. Operational Units (OTUs) were clustered with 97\% similarity cutoff using UPARSE (version 7.1 http://drive5.com/uparse/) and chimeric sequences were identified and removed using UCHIME. The taxonomy of each 16S rRNA gene sequence was analyzed by RDP Classifier (http://rdp.cme.msu.edu/) against the silva (SSU123)16S rRNA database using confidence threshold of 0.7 [13].

\section{RESULTS AND DISCUSSION}

\section{A. Characteristics of Sample Sequence Reads}

The sequencing of the amplicons pool resulted in 22,394 paired-end sequence reads, which were reduced to 17,792 assembled reads after index-based demultiplexing and assembly. The average reads per sample was 5930 and the average length was 484 bp. Rarefaction analysis indicated that there was satisfactory coverage for all of the samples (ESC: 96\% 97\%, Similarity level 0.03). The total number of OTU was 1010 and the richness of samples R12, R9 and R5 was 451,436 and 361 OTUs. The Good's coverage for 16S DNA amplicons and community richness estimator for samples R12, R9 and R5 are shown in Table I. Phylogenetic diversity in samples R12, R9 and R5 using the Shannon index show the following pattern: R12> R9> R5.

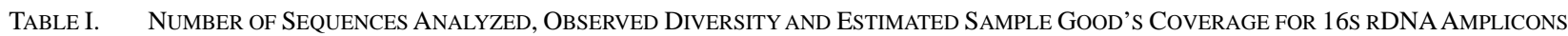
ANALYZED

\begin{tabular}{ccccccc}
\hline \multirow{2}{*}{ Sample ID } & \multirow{2}{*}{ Reads } & \multicolumn{5}{c}{ Similarity level 0.03 } \\
\cline { 3 - 7 } & & OTU & ace & chao & coverage & shannon \\
\hline \multirow{2}{*}{ R12 } & \multirow{2}{*}{5423} & 451 & 1050 & 784 & 0.96 & 3.22 \\
& & & $(951,1169)$ & $(684,927)$ & & $(3.15,3.28)$ \\
R5 & \multirow{2}{*}{4666} & \multirow{2}{*}{361} & 577 & 558 & 0.97 & 2.73 \\
& & & $(516,661)$ & $(492,658)$ & & $(2.67,2.78)$ \\
R9 & \multirow{2}{*}{7451} & 436 & 853 & 661 & 0.97 & 3.07 \\
& & & $(776,947)$ & $(591,763)$ & & $(3.0,3.13)$ \\
\hline
\end{tabular}




\section{B. Taxonomical Assessment}

An overview of the OTUs is shown in Fig. 1. At the genera level, the suanrou samples contained Staphylococcus, Lactobacillus, Cyanobacteria, Tetragenococcus, Weissella, Rickettsiales and Brevibacterium. Rare taxonomies were grouped as "other". The dominant group in R12 was Staphylococcus (74.88\%), unclassified Cyanobacterium (9.48\%) and Tetragenococcus (5.55\%). Minor groups included Weissella (1.49\%), unclassified Rickettsiales (1.31\%) and no-rank Rhodobacteraceae (1\%). Groups of no-rank Cyanobacterium (0.72\%), Lactobacillus (0.5\%) and Brevibacterium (0.04\%) were the least abundant.
Staphylococcus (96.64\%) was also the predominant microbial group in R5, Brevibacterium (1.44\%) was less abundant, and the groups of no-rank Weissella $(0.36 \%)$, no-rank Rhodobacteraceae (0.24\%), unclassified Cyanobacterium (0.09\%), no-rank Cyanobacterium $(0.06 \%)$ and Corynebacterium (0.04\%) were also detected. A different microbial community profile was found in sample R9.The proportions of genera in R9 were: Lactobacillus (88.18\%), Weissella (5.07\%), Staphylococcus (1.58\%), unclassified Cyanobacterium (0.85\%), no-rank Rhodobacteraceae (0.35\%), no-rank Cyanobacterium (0.26\%), unclassified Rickettsiales (0.08\%), Tetragenococcus $(0.07 \%)$ and Corynebacterium $(0.01 \%)$.

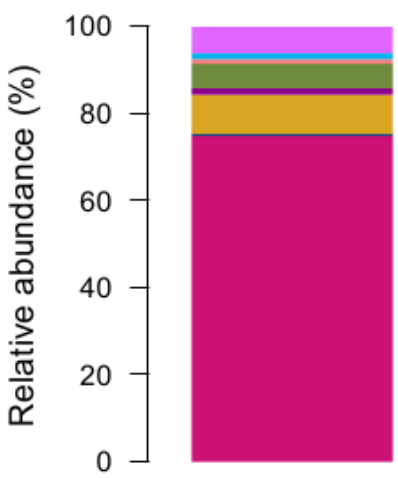

R12

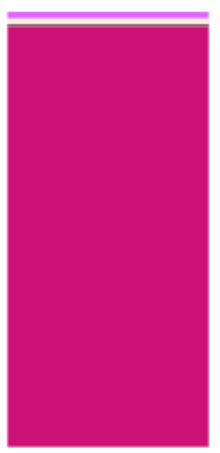

R5

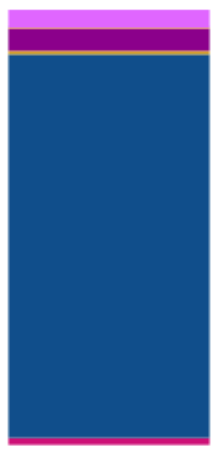

R9

$\begin{array}{lll}\square \text { Staphylococcus } & \square \text { Weissella } & \square \text { unclassified_Rickettsiales } \\ \square \text { Lactobacillus } & \square \text { Tetragenococcus } & \square \text { Brevibacterium } \\ \square \text { unclassified_Cyanobacteria } & \square \text { no_rank_Cyanobacteria } & \square \text { Others }\end{array}$

Figure 1. Bacterial composition of the R12, R5 and R9 production

In Fig. 2, a Venn plot was used to indicate common and unique OTUs present in samples R12, R9 and R5. Only 29 sequences were common across all samples $(2.87 \%$ of 1010 OTUs) and comparatively, the Venn plot confirms that the community profile of R9 is different from the community profiles of samples R12 and R5.

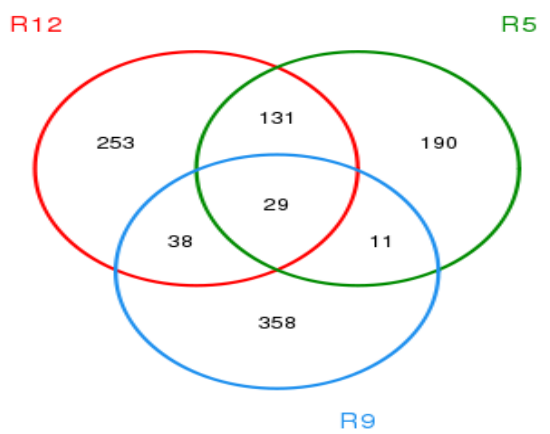

Figure 2. OTU Venn analysis of sample R12, R5 and R9 (3\% distance level).

Unique objects: All=1010; R12=451; R5=361; R9=436

\section{Taxonomical Structure}

Classical microbiology identified the genera of Staphylococci and Lactobacillus as the dominant flora in Suanrou [2, 3, 4]. In our study, a detailed analysis of the bacterial community in Suanrou using HTS confirms this observation. In addition to these dominant groups, the taxonomical analysis carried out on OTUs obtained with 454- pyrosequencing also shows a complex community profile. This complex community profile is a gateway to developing indigenous starter cultures [12]. The main dominant bacteria strains have been isolated and identified in another study of our group. A total of 56 strains were isolated in which 13 isolates were identified. Strain of Lactobacillus curvatus has been described as species with great antioxidant properties [14]. Strains of Staphylococcus sp. 303402, Lactobacillus kimchi and Lactobacillus graminis have been screened and confirmed as exopolysaccharides-producing LAB with antioxidant properties [15].

Microbial diversity in Suanrou was also dependent on fermenting time. Sample R12 was fermented for 6 months before sampling and has the highest bacterial diversity 
when compared to sample R9 (3 months) samples R5 (1 month). We observed that the bacterial distribution of R9 was significantly different to the other two samples because Lactobacillus was predominant. In comparing the recipe for Suanrou in these samples, R9 contained only $7 \%$ added salt while R12 and R5 contained 15\% added salt and added salt could be a significant factor in the development of the microbial community.

\section{Specific Fermentation Microorganisms}

LAB, coagulase-negative Staphylococcaceas (CNS) and coagulase-negative cocci (CNC) are the main groups of bacteria that are considered technologically important during fermentation and ripening of fermented meat products [9, 12, 16]. Staphylococcus contribute to the development of color and flavor in fermented meat products mainly by degrading free amino acids and inhibiting the oxidation of unsaturated free fatty acids [12]. LAB is an essential agent during meat fermentation improving the hygienic and sensory quality of the final product. Its fermentative metabolism prevents the development of spoilage and pathogenic microflora by acidification of the product, also contributing to its color stabilization and texture improvement [16].

In this study, we demonstrated that the two most important genera involved in Suanrou products were Staphylococcus and Lactobacillus and in addition HTS also identified the genus of Weissellaand Tetragenococcus (Fig.1) as important groups in Suanrou products. Weissella species is ubiquitous in the natural environment in which fresh and fermented foods are prepared (cereal foods, sea foods, cassava, sourdough, Soy sauce, Kava beverages, Kimchi and Stinky tofu) [7]. Recently, a novel bacteriocin-producing strain, $W$. hellenica D1501, was isolated from Chinese sour meat product which exhibits a broad range of inhibitory activity against foodborne bacteria and some molds and yeasts [1]. Tetragenococcus, is known to survive in extreme salt environments, and are frequently found in fermented foods, such as Doen jang, fish sauce, soy sauce and raw fermented sausage [7]. Genus of Tetragenococcus has been reported in association with osmotic tolerance and acid tolerance and it was also suggested as a starter for fermented meat products $[17,18]$.

The HTS of 16S rRNA amplicons also identified a group of unclassified Cyanobacteriain sample R12 (9.48\%) which are also present small amounts in R9 and R5. It is not known whether Cyanobacteria and the other groups are natural contaminants specific to location of production and further research should try to identify the roles of these groups.

\section{CONCLUSIONS}

The bacterial community distribution involved in food fermentations is one of the most important factors shaping the final characteristics of traditional fermented foods. Here, we investigated the bacterial community structure and identified the main microorganisms of traditional sour meat products of China by the introduction of HTS methods. The results revealed that the main taxonomic groups of sour meat contain genera of Staphylococcus, Lactobacillus, Cyanobacterium, Tetragenococcus, Weissella, Rickettsiales and Brevibacterium, of which the Staphylococci and Lactobacillus are the overwhelmingly predominant population. The results indicated that microbial diversity in sour meat is comparative high, while the specific fermentation microorganism is particularly prominent. It is helpful to develop indigenous starters for fermented meat products.

\section{ACKNOWLEDGEMENT}

This work was supported by grants from National Natural Science Foundation of China (31260379).

\section{REFERENCES}

[1] Chen, C., Chen, X. H., Jiang, M., Rui, X., Li, W. and Dong, M. S.,”A newly discovered bacteriocin from Weissella hellenica D1501 associated with Chinese Dong fermented meat (NanxWudl)”, Food Control, vol. 42, pp. 116-124, 2014.

[2] Li, Z. J. and Jiang, H. H, "Alteration of Microbes Flora of Chinese Traditional Sour Meat during Fermentation", Microbiol. China, vol. 31, pp. 9-13, 2004 (In Chinese).

[3] Li, Z. J., "Isolation and Identification of Staphylococci in Chinese Traditional Cured Meat and its Application”, Biotech. Bulletin, vol. 3, pp. 77-81, 2006 (In Chinese).

[4] Che, K., Ma, C. G., Huang, Q. and Yu, J., "Isolation and identification of the lactic acid bacteria in Xiangxi traditional fermented meat", China. Brewing, vol. 188, pp. 25-28, 2008 (In Chinese).

[5] Luca, C., Paola, d. andKalliopi, R., "Biodiversity and dynamics of meat fermentations: The contribution of molecular methods for a better comprehension of a complex ecosystem”, MeatSci., vol. 89, pp. 296-302, 2011.

[6] Cocolin, L., Manzano, M., Cantoni, C. andComi, G., "Denaturing gradient gel electrophoresis analysis of the 16S rRNA gene V1 region to monitor dynamic changes in the bacterial population during fermentation of Italian products”, Appl. Envirn. Microbiol., vol. 67, pp. 51135121, 2001.

[7] Luca, C., Valentina, A., Paola, D., Roberta, G. andKalliopi, R., "Culture independent methods to assess the diversity and dynamics of microbiota during food fermentation”, Int. J. Food Microbiol., vol. 167, pp. 29-43, 2013.

[8] Ercolini, D., De Filippis, F., La Storia, A. and Lacono, M., “'Remake’ by high throughput sequencing of the microbiota involved in the production of water buffalo mozzarella cheese”, Appl. Environ. Microbiol., vol. 78, pp. 8142-8145, 2012.

[9] Anna, G., Ilario, F., Antonietta, L. S., Kalliopi, R., Danilo, E. and Luca, C., "Monitoring of the microbiota of fermented products by culture independent rRNA-based approaches", Int. J. Food Microbiol., vol. 212, pp. 67-75, 2015.

[10] Justyna, P., Annalisa, R., Vincenza, P., Lorenzo, M. and Edoardo, P., "Bacterial diversity in typical Italian salami at different ripening stages as revealed by high- throughput sequencing of 16S rRNA amplicons”, Food Microbiol., vol. 46, pp. 342-356, 2015.

[11] Annalisa, R., Vincenza, P., Francesco, M., Justyna, P., Irene, F., Lorenzo, M. and Edoardo, P., "High-throughput assessment of bacterial ecology in hog, cow and ovine casings used in products production”, Int. J. Food Microbiol., vol. 212, pp. 49-59, 2015.

[12] Talon, R., Leroy, S. and Lebert, I., "Microbial ecosystems of traditional fermented meatproducts: The importance of indigenous starters”, Meat Sci., vol. 77, pp. 55-62, 2007.

[13] Katherine R Amato, Carl J Yeoman, Angela Kent, Nicoletta Righini,et al., "Habitat degradation impacts black howler monkey (Alouattapigra) gastrointestinal microbiomes", The ISME J.,vol. 7, pp. 1344-1353, 2013. 
[14] Zhan, J. L., Wang, Y. F., Hu, P., "Identification and Characterization of Lactobacillus curvatus LAB26 with Antioxidant Potential from Sour Meat of the Dong Minority”, Meat Research, vol. 28, pp. 1-4, 2014 (In Chinese).

[15] Zhang, Y. L., Hu, P., Wang, J. L., Liao, Q. W., "Isolation of exopolysaccharides-producing lactic acid bacteria and its antioxidant properties”, China brewing, vol. 34, pp. 37-43, 2015 (In Chinese).

[16] Fadda, S., López, C. and Vignolo, G., "Role of lactic acid bacteria during meat conditioning and fermentation: Peptides generated as sensorial and hygienic biomarkers”, Meat Sci., vol. 86, pp. 66-79,
2010.

[17] Amadoro, C., Rossi, F., Piccirilli, M. and Colavita, G., "Tetragenococcus koreensisis part of the microbiota in a traditional Italian raw fermented sausage”, Food Microbiol., vol. 50, pp. 78-82, 2015.

[18] He, G. Q., Wu, C. D., Huang, J. and Zhou, R. Q., “Acid tolerance response of Tetragenococcus halophilus: A combined physiological and proteomic analysis”, Process Biochem., vol. 51, pp. 213-219, 2016. 